EPJ Web of Conferences 66, 02051 (2014)

DOI: $10.1051 /$ epjconf/ 20146602051

(C) Owned by the authors, published by EDP Sciences, 2014

\title{
Application of the generator coordinate method to neutron-rich Se and Ge isotopes
}

\author{
Koji Higashiyama ${ }^{1, a}$ and Naotaka Yoshinaga ${ }^{2}$ \\ ${ }^{1}$ Department of Physics, Chiba Institute of Technology, Narashino, Chiba 275-0023, Japan \\ ${ }^{2}$ Department of Physics, Saitama University, Saitama City 338-8570, Japan
}

\begin{abstract}
The quantum-number projected generator coordinate method (GCM) is applied to the neutron-rich Se and Ge isotopes, where the monopole and quadrupole pairing plus quadrupole-quadrupole interaction is employed as an effective interaction. The energy spectra obtained by the GCM are compared to both the shell model results and the experimental data. The GCM reproduces well the energy levels of high-spin states as well as the low-lying states. The structure of the low-lying collective states is analyzed through the GCM wave functions.
\end{abstract}

\section{Introduction}

The intriguing properties of the even-even Se and Ge isotopes in the mass region $A \sim 80$ have been investigated in a number of previous experimental and theoretical studies [1-3]. These isotopes belong to a typical transitional region that lies between spherical and deformed regions. The structure of their low-lying states can be attributed to the interplay of rotational and vibrational collective motions. For high-spin states, $\gamma$-ray spectroscopy of the near-yrast states in the $N=44$ and 46 isotones of Se $\left({ }^{80,82} \mathrm{Se}\right)$ was carried out for deep-inelastic reactions [4].

Recently, full-fledged shell-model calculations were performed on the even-even and odd-mass nuclei in this mass region [5]. The calculations reproduced well the experimental energy levels and electromagnetic transition rates for both the low-lying and high-spin states. In order to investigate the features of these states, the energy spectra in the shell model were compared with those in a pair-truncated shell model (PTSM). In the model, the full shell model space is truncated within the subspace where the collective nucleon pairs with various angular momenta are assumed to be its building blocks. The same set of the interactions as employed in the shell model calculations was applied to even-even nuclei, and an excellent agreement with the shell model result was achieved for energy levels. Through the analysis of the PTSM wave functions, it was found that the angular momenta zero and two collective pairs are dominant in low-lying states, while the effect of the alignment of two $0 g_{9 / 2}$ neutrons becomes apparent above $8_{1}^{+}$states.

In the present study, we apply the quantum-number-projected generator coordinate method (GCM) to ${ }^{78} \mathrm{Ge}$ under the same interaction as used in the previous shell model study [5]. We do not discuss other nuclei, but similar results are obtained for ${ }^{76} \mathrm{Ge},{ }^{80} \mathrm{Se}$, and ${ }^{78} \mathrm{Se}$. Their results and the detailed prescriptions for the GCM will be presented in a forthcoming paper [6].

ae-mail: koji.higashiyama@it-chiba.ac.jp 


\section{Theoretical framework}

The previous GCM studies [7,8] made it clear that, for a description of the nuclear collective and single-particle motions in a transitional region, the angular momenta of the neutron and proton systems $\left(I_{v}\right.$ and $\left.I_{\pi}\right)$ should be projected out separately, and the total spin $I$ is constructed by angular momentum coupling. Thus in the present GCM calculations, the angular momentum projection is performed separately in each proton or neutron space. To generate functions for the GCM, we employ the intrinsic states $\left|\Phi_{\tau}(\beta, \gamma)\right\rangle$ for either neutron or proton system $(\tau=v$ or $\pi)$, where $\beta$ and $\gamma$ indicate axial and triaxial quadrupole deformations, respectively. The $\rho$ th GCM wave function with angular momentum $I_{\tau}$ in either neutron or proton space is given by

$$
\left|\Psi_{I_{\tau} M_{\tau} \rho}^{(\tau)}\right\rangle=\sum_{i} \sum_{K_{\tau}=-I_{\tau}}^{I_{\tau}} \mathcal{F}_{K_{\tau} \rho}^{I_{\tau} i} \hat{P}_{M_{\tau} K_{\tau}}^{I_{\tau}}\left|\Phi_{\tau}\left(\beta_{i}, \gamma_{i}\right)\right\rangle,
$$

where $\hat{P}_{M_{\tau} K_{\tau}}^{I_{\tau}}$ is the spin projection operator, $\mathcal{F}_{K_{\tau} \rho}^{I_{\tau} i}$ represents the weight function to be determined by solving the Hill-Wheeler equation, and $i$ stands for a representative point with deformation $(\beta, \gamma)$. Then, the many-body wave function for an even-even nucleus can be written as

$$
\left|\Psi_{I M}\left(I_{\nu} \rho I_{\pi} \sigma\right)\right\rangle=\left[\left|\Psi_{I_{\nu} \rho}^{(v)}\right\rangle \otimes\left|\Psi_{I_{\pi} \sigma}^{(\pi)}\right\rangle\right]_{M}^{(I)}
$$

where $I$ is the total spin and $M$ is its projection. The intrinsic state $\left|\Phi_{\tau}(\beta, \gamma)\right\rangle$ is constructed by the following procedure. First we consider the intrinsic Nilsson hamiltonian for either neutron or proton space:

$$
\hat{h}_{\tau}=\sum_{j m} \varepsilon_{j} c_{j m \tau}^{\dagger} c_{j m \tau}-\hbar \omega \beta\left[\cos \gamma \hat{Q}_{0 \tau}-\frac{\sin \gamma}{\sqrt{2}}\left(\hat{Q}_{2 \tau}+\hat{Q}_{-2 \tau}\right)\right]
$$

with

$$
\hat{Q}_{\mu \tau}=\sum_{j m, j^{\prime} m^{\prime}}\left\langle j m \tau\left|r^{2} Y_{\mu}^{(2)}\right| j^{\prime} m^{\prime} \tau\right\rangle c_{j m \tau}^{\dagger} c_{j^{\prime} m^{\prime} \tau}
$$

The intrinsic single-particle deformed state $|\alpha \tau\rangle=b_{\alpha \tau}^{\dagger}|-\rangle$ is related with the spherical basis state $|j m \tau\rangle=c_{j m \tau}^{\dagger}|-\rangle$ as

$$
b_{\alpha \tau}^{\dagger}=\sum_{j m} F_{j m, \alpha \tau} c_{j m \tau}^{\dagger},
$$

by diagonalizing the Nilsson hamiltonian $\hat{h}_{\tau}$. In order to include the pairing correlation, we introduce the nucleon pair creation operator as

$$
\Lambda_{\tau}^{\dagger}(\beta, \gamma)=\sum_{\alpha>0} f_{\alpha \tau}(\beta, \gamma) b_{\alpha \tau}^{\dagger} b_{\bar{\alpha} \tau}^{\dagger}
$$

where $\bar{\alpha}$ indicates the time reversal state of $\alpha$. Then the intrinsic many-body states for neutrons or protons are written as

$$
\left|\Phi_{\tau}(\beta, \gamma)\right\rangle=\mathcal{N}\left[\Lambda_{\tau}^{\dagger}(\beta, \gamma)\right]^{N / 2}|-\rangle
$$

where $\mathcal{N}$ denotes the normalization. Then, the structure coefficients $f$ 's are determined by variation for a definite deformation $(\beta, \gamma)$ :

$$
\delta\left\langle\Phi_{\tau}(\beta, \gamma)\left|\hat{H}_{\tau}\right| \Phi_{\tau}(\beta, \gamma)\right\rangle=0 .
$$

Here, $\hat{H}_{\tau}$ is the spherical Hamiltonian between like nucleons $(\tau=v$ or $\pi)$. The potential energy surface (PES) is defined as

$$
E_{\tau}(\beta, \gamma)=\left\langle\Phi_{\tau}(\beta, \gamma)\left|\hat{H}_{\tau}\right| \Phi_{\tau}(\beta, \gamma)\right\rangle
$$



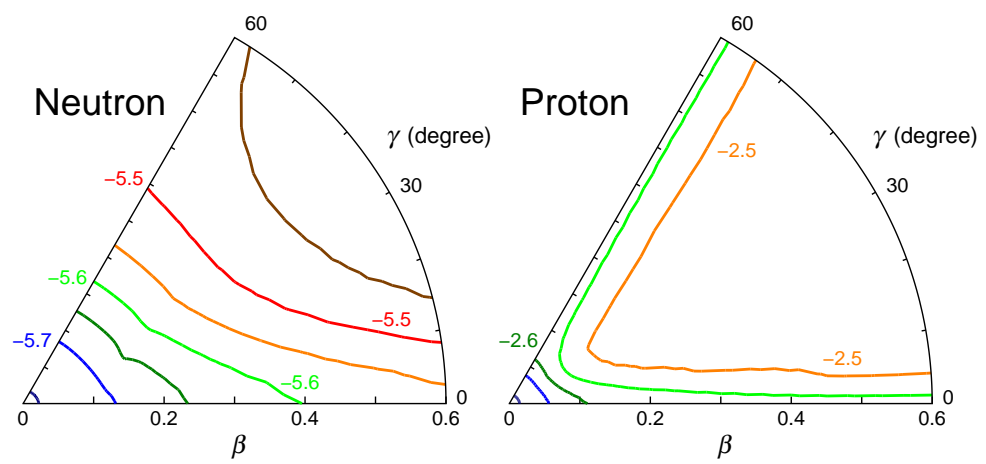

Figure 1. The contour plot of the PES calculated for neutrons (left panel) and protons (right panel) in ${ }^{78} \mathrm{Ge}$. The contour line separation is $0.05 \mathrm{MeV}$.

\section{Numerical results}

In describing the nuclei in the mass $A \sim 80$ region, valence neutron holes and proton particles occupy all the four orbitals, $0 g_{9 / 2}, 1 p_{1 / 2}, 1 p_{3 / 2}$, and $0 f_{5 / 2}$, in the major shell between magic numbers 28 and 50. The effective Hamiltonian consists of the single-particle energies, the monopole and quadrupole pairing plus quadrupole quadrupole interactions:

$$
H=\sum_{j m \tau} \varepsilon_{j \tau} c_{j m \tau}^{\dagger} c_{j m \tau}-\sum_{\tau}\left[G_{0 \tau} P_{\tau}^{\dagger(0)} P_{\tau}^{(0)}+G_{2 \tau} P_{\tau}^{\dagger(2)} \tilde{P}_{\tau}^{(2)}+\kappa_{\tau}: Q_{\tau} \cdot Q_{\tau}:\right]-\kappa_{v \pi} Q_{v} \cdot Q_{\pi}
$$

The strengths of the effective interactions are determined so as to reproduce the even-even nuclei with mass $A \sim 80$. All other details are given in Ref. [5].

In Fig. 1, the contour plots of the PES in Eq. (9) are shown in neutron and proton spaces for ${ }^{78} \mathrm{Ge}$. For both neutron and proton spaces, triaxiality appears, but it is subtle for proton space. The potential surfaces are very shallow and have no definite minima. The GCM calculations are carried out in two cases: (i) triaxial deformations (20 points) with $\beta=0.10,0.20,0.30, \gamma=10^{\circ}, 20^{\circ}, 30^{\circ}, 40^{\circ}, 50^{\circ}$, and $(\beta, \gamma)=\left(0.40,20^{\circ}\right),\left(0.40,40^{\circ}\right),\left(0.50,10^{\circ}\right),\left(0.50,30^{\circ}\right),\left(0.50,50^{\circ}\right)$; (ii) only axial deformations $(31$ points) with $\beta=0.00,0.04,0.08, \cdots, 0.60$ and $\gamma=0^{\circ}, 60^{\circ}$. In Fig. 2 energy spectra are compared with experimental results, shell model, GCM (triaxial) and GCM (axial), respectively. In both cases of triaxial and axial deformations, the GCM reproduces the energy levels of the even-spin yrast band well. For excited states, the GCM calculations performed by assuming triaxial deformations are in good agreement with the shell model results, especially for the $2_{2}^{+}, 3_{1}^{+}, 4_{2}^{+}$, and $5_{1}^{+}$states, which are members of the quasi- $\gamma$ band. However, the energy levels calculated by assuming only axial deformations (the $2_{2}^{+}, 3_{1}^{+}$, and $5_{1}^{+}$states) are higher compared to the shell model results. Apparently, the description of the $2_{2}^{+}, 3_{1}^{+}, 4_{2}^{+}$, and $5_{1}^{+}$states is not satisfactory when assuming only the axially symmetric shape. The triaxial components play an essential role in the description of these states.

The ground state energies for the ${ }^{78} \mathrm{Ge}$ are $E_{\mathrm{SM}}=-11.0530 \mathrm{MeV}, E_{\mathrm{GCM} \text { (triaxial) }}=-11.0428 \mathrm{MeV}$, and $E_{\mathrm{GCM}(\text { axial })}=-10.9643 \mathrm{MeV}$, respectively for the SM, the GCM with triaxial deformations and the GCM only with axial deformations. A large improvement is seen assuming the triaxial shape for the ground state energy. 


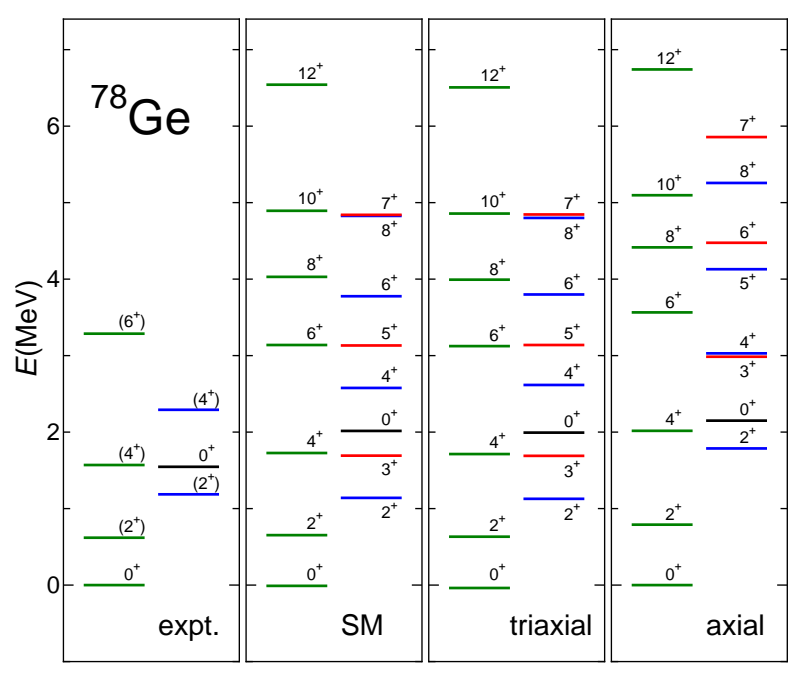

Figure 2. Energy levels of the yrast and quasi- $\gamma$ bands in ${ }^{78} \mathrm{Ge}$. From the left, energy levels in experiment (expt.), the shell model (SM), the triaxial GCM (triaxial), and the axial GCM (axial) are displayed.

\section{Summary}

In this paper the GCM is applied to the neutron-rich Se and Ge isotopes. In the present scheme, the proton and neutron parts are treated separately in the GCM stage, and they are coupled through the diagonalization of the total Hamiltonian. This is an especially important treatment for transitional nuclei and neutron rich nuclei. The energy spectra calculated by assuming axial and triaxial deformations are compared to the shell model results. In both cases, the GCM reproduces well the energy levels of the even-spin yrast band. However, the GCM results for axial deformations are not satisfactory enough to reproduce the energy levels of the quasi- $\gamma$ band. Taking account of triaxial deformations is essentially important to describe the quasi- $\gamma$ band. The PES is found to be shallow in the $\gamma$ direction and it shows $\gamma$-unstable nature or the $\mathrm{O}(6)$ like picture in the interacting boson model [9].

\section{References}

[1] K.-H. Speidel et al., Phys. Rev. C 57, 2181 (1998), and references therein.

[2] Y. Toh et al., Phys. Rev. C 87, 041304(R) (2013).

[3] N. Yoshinaga and K. Higashiyama, J. Phys. Conference Series 445, 012032 (2013).

[4] G. A. Jones et al., Phys. Rev. C 76, 054317 (2007).

[5] N. Yoshinaga, K. Higashiyama, and P. H. Regan, Phys. Rev. C 78, 044320 (2008).

[6] K. Higashiyama, and N. Yoshinaga, to be published.

[7] K. Enami, K. Tanabe, and N. Yoshinaga, Phys. Rev. C 65, 064308 (2002).

[8] K. Enami, K. Higashiyama, K. Tanabe, and N. Yoshinaga, Phys. Rev. C 66, 047301 (2002).

[9] F. Iachello and A. Arima, The Interacting Boson Model (Cambridge University Press, Cambridge, 1987). 